\title{
Les modèles de \\ livraison lors d'un achat en ligne dans le luxe
}

\section{Analyse socio-sémiotique}

Nathalie Veg-Sala*

La livraison constituant l'un des freins majeurs pour les consommateurs de produits de luxe lors d'un achat en ligne, l'objectif de cet article est d'étudier comment celle-ci pourrait être définie et mise en œuvre afin de correspondre aux valeurs de ce secteur et de satisfaire ses consommateurs. En s'appuyant sur les logiques de consommation du luxe et les composantes fonctionnelle et expérientielle de la logistique, mais aussi sur une double méthodologie socio-sémiotique, cette recherche met en évidence quatre modèles de livraison souhaités par les consommateurs (discrète, théâtralisée, secrète et normée). Des contributions théoriques et managériales en sont déduites.

* Laboratoire CEROS, Université Paris Nanterre 
$\mathrm{L}$

e marché mondial du luxe enregistre de nouveaux records de croissance. Le cabinet Bain \& Company $(2018)^{1}$ a estimé une progression de $6 \%$ du secteur des produits personnels de luxe atteignant 260 milliards d'euros. Parmi les facteurs de développement, l'étude met en évidence une accélération du e-commerce avec une croissance de $22 \%$ en 2018, représentant près de 27 milliards d'euros. Et les marques de luxe espèrent que ce mode de distribution reste encore un levier de croissance exceptionnelle dans les années à venir. Pourtant, ces mêmes marques ont longtemps hésité avant de se lancer dans la vente en ligne. L'intégration du e-commerce dans la politique de distribution constituait en effet un challenge délicat (Okonkwo, 2009) qui pouvait renforcer le paradoxe de la gestion des marques de luxe entre, d'une part, les valeurs de sélectivité, d'exclusivité et de rareté relatives au secteur (Catry, 2007) et, d'autre part, la diffusion massive, concomitante à la vente en ligne (Roux et Floch, 1996 ; Veg-Sala et Geerts, 2015b). Cette crainte avait été constatée par Dall'Olmo Riley et Lacroix (2003) qui montraient, au début des années 2000, que seuls $15 \%$ des marques de luxe proposaient la vente en ligne (sur 54 sites étudiés). Progressivement, cette proportion a augmenté, passant à $41,8 \%$ en 2011, à $52 \%$ en 2014 et à $70,2 \%$ en $2018^{2}$. Mais si quasiment toutes les marques de luxe proposent aujourd'hui la vente en ligne, les achats sur Internet des consommateurs ne représentent que $10 \%$ des ventes. Comment expliquer ce décalage ?

Le manque d'expérience perçue par les consommateurs de luxe lors d'un achat en ligne serait en cause. Certains clients craignent de ne pas ressentir l'ambiance prestigieuse des magasins, de ne pas être suffisamment dans l'hédonisme, la sensorialité ou encore l'interaction. Ces déficits d'expérience sont évoqués pour l'ensemble $\mathrm{du}$ processus d'achat en ligne, de la navigation sur le site jusqu'à la livraison des produits (Veg-Sala et Geerts, 2015a). À cela, s'ajoutent des craintes de ne pas recevoir leur commande ou de la recevoir en mauvais état (Veg-Sala et Geerts, 2015b). Quelles sont les attentes particulières des consommateurs vis-à-vis de la livraison des produits des marques de luxe ? Dans les recherches en marketing, la question de la livraison a très peu été abordée. Elle l'est, parfois, dans les travaux portant sur la qualité de service en ligne (Wolfinbarger et Gilly, 2003 ; Parasuraman et al., 2005 ; Bauer et al., 2006 ; Ha et Stoel, 2009) mais uniquement en termes de temps ou de coûts. De plus, il s'agit de recherches qui se focalisent sur la grande consommation et qui ne prennent donc pas en compte les spécificités du secteur du luxe.

La question de la livraison est davantage étudiée dans les recherches en logistique qui s'intéressent à l'ensemble du processus d'acheminement des produits, de leur fabrication jusqu'aux clients (Fulconis

\footnotetext{
1. Bain \& Company (2018). «Luxury Goods Worldwide Market Study », Fall-Winter https://www.bain.com/ contentassets/8df501b9f8d6442eba00040246c6b4f9/bain_digest_luxury_goods_worldwide_market_study_fall_winter_2018.pdf

2. Etude ad hoc menée sur 74 marques de bien personnels de luxe (comprenant les marques de l'article de Dall'Olmo Riley et Lacroix (2003), complétées par d'autres marques du Comité Colbert ou des marques appartenant aux principaux groupes de luxe (LVMH, Kering, Richemont).
} 
et al., 2011 ; Baron, 2014) et qui soulignent une évolution des fonctions logisticiennes, allant vers plus de services (Badot et Paché, 2007 ; Ducret, 2012 ; Rouquet et Paché, 2017). Le développement stratégique de la logistique et de ses métiers fait écho aux recherches de Granzin et Bahn (1989) qui suggéraient, déjà en leur temps, le besoin d'établir plus de lien entre la logistique et le marketing. Ils insistaient sur la nécessité d'orienter la logistique comme un élément de satisfaction des consommateurs. Et proposaient (ibid., 1989) de revoir les tâches logistiques en fonction de la nature des situations de consommation en faisant la distinction entre les produits à caractère tangible et ceux à caractère intangible. Les fonctions logistiques devraient donc être adaptées selon le secteur (Ducret, 2012).

Toutefois, à notre connaissance, aucune recherche n'a étudié la question de la livraison dans le domaine du luxe, que ce soit en marketing ou en logistique. Mais, plusieurs articles et ouvrages spécialisés mettent en avant le fait que la gestion des marques de luxe est différente et que les valeurs propres au luxe de : sélectivité, rareté et exclusivité contraignent les maisons de luxe à adapter les stratégies habituellement utilisées par les marques d'autres secteurs (Roux et Floch, 1996 ; Okonkwo, 2009 ; Veg-Sala et Geerts, 2015a, 2015b ; Merk et Michel, 2019).

L'objectif de cet article est d'étudier comment la livraison dans le cadre de la vente en ligne de produits de marques de luxe peut être définie et mise en œuvre afin de correspondre aux valeurs de ce secteur et de satisfaire ses consommateurs. Pour ce faire, la recherche s'appuie sur la théorie de la dualité du luxe (Vigneron et Johnson,
1999 ; Kastanakis et Balabanis, 2012). Celle-ci différencie les consommateurs de luxe selon des logiques de consommation personnelle ou interpersonnelle afin de mieux appréhender leurs attentes. L'article prend en considération les travaux sur la qualité de service en ligne (Parasuraman et al., 2005 ; Collier et Bienstock, 2006 ; Cristobal et al., 2007 ; Bauer et al., 2006) mais intègre également les travaux en logistique qui distinguent les composantes fonctionnelles et expérientielles de celle-ci (Badot et Paché, 2007 ; Rouquet et Paché, 2017).

Puis, une double méthodologie qualitative est mise en œuvre. Elle s'appuie sur une approche socio-sémiotique afin de mieux appréhender les significations des discours des consommateurs et des marques (Courtès, 1991 ; Floch, 1990). Deux études sont menées : 1) une étude qualitative avec des entretiens auprès de consommateurs de luxe et 2) une étude sémiotique visuelle structurale sur des marques de luxe et leurs sites Internet. La confrontation des attentes des consommateurs de luxe et des stratégies développées par les marques permet d'apprécier particulièrement les écarts et d'en déduire des contributions théoriques et managériales.

\section{I - LE LUXE ET LA THÉORIE DE LA DUALITÉ DES LOGIQUES DE CONSOMMATION}

Malgré le peu de consensus sur la définition du luxe, les chercheurs s'accordent à dire qu'il s'agit d'un concept subjectif et multidimensionnel couvrant un large éventail de perceptions des consommateurs (Wiedmann et al., 2007). Son sens a évolué dans le temps, passant principalement d'une 
vision interpersonnelle (entre les années 1950 et 1990) à une vision plus personnelle (à partir des années 1990), pour, finalement, aboutir à une dualité entre ces approches (Roux et Floch, 1996 ; Lipovetsky et Roux, 2003).

\section{Le luxe interpersonnel}

Traditionnellement, le luxe a longtemps été associé à des motivations sociales. Acquérir des produits de marques de luxe avait pour principal objectif d'impressionner les autres, impliquant l'influence sociale des personnes significatives et leur impact sur leurs opinions, leurs perceptions et leurs comportements (Vigneron et Johnson, 1999 ; Shukla et Purani, 2012 ; Roux et al., 2017). Le luxe interpersonnel est un marqueur social. Dans cette logique de consommation, le luxe recrée une stratifica-tion sociale (Bastien et Kapferer, 2008) et n'existe que s'il permet d'être montré. Consommer des produits de luxe selon la logique interpersonnelle a pour fonction de se différencier des autres, de montrer une distinction, un statut social supérieur (Bourdieu, 1979).

\section{2. Évolution de la logique de consommation et développement du luxe personnel}

La vision interpersonnelle du luxe a ensuite évolué, faisant émerger, à partir des années 1990, une autre approche : celle du luxe personnel. Celui-ci place les sentiments et les émotions au centre de la consommation du luxe, au-delà d'un désir de démonstration sociale (Vigneron et Johnson, 1999 ; Shukla et Purani, 2012). L'hédonisme prime sur la fonctionnalité. Le luxe personnel se vit davantage qu'il ne se consomme (Bastien et Kapferer, 2008). Plus spécifiquement, l'orientation personnelle du luxe est associée à la recherche d'un plaisir individuel (avantages affectifs personnels), permettant d'exprimer sa personnalité (avantages symboliques personnels), en correspondant à ses attitudes et goûts (avantages utilitaires personnels) (Wong et Ahuvia, 1998).

\section{Dualité du luxe et gestion des marques de luxe}

Au-delà de l'opposition entre le luxe personnel et le luxe interpersonnel, des chercheurs ont montré que ces deux logiques de consommation devraient être prises en compte dans la gestion marketing des marques de luxe (Wong et Ahuvia, 1998 ; Vigneron et Johnson, 1999 ; Roux et al., 2017). L'échelle de mesure «Brand Luxury Indice » (BLI) reflète cette bidimensionnalité et prend en compte les deux composantes du luxe, agrégeant cinq sous-échelles (faisant référence, pour le « Luxe interpersonnel », à la remarquabilité perçue, à l'unicité et à la qualité et, pour le « Luxe personnel », à l'hédonisme et l'extension de soi) (Vigneron et Johnson, 2004).

Il découle de cette théorie qu'un produit ou un service de luxe, si l'on souhaite qu'il rencontre un vrai succès économique durable, doit impérativement posséder les deux aspects du luxe : interpersonnel (luxe comme affirmation sociale vis-à-vis des autres) et personnel (luxe comme plaisir individuel pour soi) (Bastien et Kapferer, 2008). Les marques doivent intégrer cette dualité dans l'ensemble de leur gestion et de leur développement. Cela pourrait être le cas au niveau de leur politique de distribution en 
ligne et, plus particulièrement, au niveau de la livraison. Toutefois, les recherches qui se sont focalisées, de près ou de loin, sur cette thématique n'ont pas intégré ces éléments liés aux logiques de consommation du luxe.

\section{II - LE LUXE ET LA LIVRAISON : VERS UNE FONCTION EXPÉRIENTIELLE}

\section{Les perceptions des consommateurs de luxe face au e-commerce et à la livraison}

Plusieurs recherches ont noté une intensification du paradoxe des marques et de leur politique de distribution via l'utilisation de la vente en ligne. L'e-commerce s'oppose aux caractéristiques de rareté subjective liée au luxe et au besoin de maintenir une sélectivité des canaux de distribution (Catry, 2007). Il renforce, entre autres, la banalisation des marques de luxe par l'accroissement potentiel des ventes et leur exposition (Okonkwo, 2009). Le paradoxe majeur tient dans la difficulté de créer une atmosphère multisensorielle et de susciter un désir d'acquisition sur un outil de diffusion de masse (Bastien et Kapferer, 2008 ; Okonkwo, 2009). L'e-commerce représente ainsi à la fois une opportunité et une menace pour les marques de luxe (Dall'Olmo Riley et Lacroix, 2003).

Cette ambivalence est largement perçue par les consommateurs de luxe dont les comportements oscillent entre des attitudes positives (plus grande facilité d'achat, informations sur les produits) et des attitudes négatives (manque de confiance, incohérence entre les valeurs du luxe et du e-commerce). Plus spécifiquement, au-delà des motivations et freins classiques liés à l'achat en ligne (Dall'Olmo Riley et Lacroix, 2003 ; Liu et al., 2013), le déficit d'expérience d'achat est particulièrement souligné dans le cas d'un achat en ligne pour des produits de marques de luxe (Veg-Sala et Geerts, 2015a). Les consommateurs mettent en avant des déficits sensoriels, d'ambiance, d'hédonisme, d'interaction et de personnalisation. Enfin, les clients des marques de luxe insistent sur les risques liés à la livraison de ce type de produits (VegSala et Geerts, 2015a).

La livraison pourrait être utile pour réduire les perceptions de déficits d'expérience. En effet, c'est lors de cette étape qu'un contact physique entre le client et la marque peut s'établir et qu'il serait possible de mettre en place des stratégies visant à répondre aux attentes des individus et ainsi améliorer la qualité de service.

\section{La livraison dans les recherches sur la qualité de service en ligne}

La qualité de service en ligne correspond à l'étendue à laquelle un site Internet facilite un magasinage efficace, l'achat et la livraison (Zeithaml, 2002). Elle joue un rôle fondamental dans le processus d'achat en ligne dans le sens où elle impacte positivement la satisfaction des consommateurs, la qualité perçue, la fidélité ainsi que l'attitude à l'égard du site Internet (Wolfinbarger et Gilly, 2003 ; Cristobal et al., 2007 ; Ha et Stoel, 2009).

Plusieurs chercheurs ont travaillé sur la conceptualisation et la mesure de la qualité de service en ligne (Zeithaml, 2002 ; Trocchia et Janda, 2003 ; Wolfinbarger et Gilly, 2003 ; Parasuraman et al., 2005 ; Collier et Bienstock, 2006 ; Cristobal et al., 2007 ; Bauer et al., 2006). À ce jour, aucun 
consensus ne ressort quant aux dimensions du concept. Plus précisément, la place réservée au processus de livraison n'est pas toujours la même. Elle n'est d'ailleurs pas prise en compte dans certaines échelles (Trocchia et Janda, 2003). Et, lorsqu'elle est intégrée, la livraison est souvent incluse dans les dimensions liées à la fiabilité et à la réalisation/finalisation de l'achat en ligne (Wolfinbarger et Gilly, 2003 ; Parasuraman et al., 2005 ; Bauer et al., 2006). Dans la plupart des cas, un à trois items sont utilisés afin d'évaluer la livraison en termes de rapidité et de délai (Wolfinbarger et Gilly, 2003 ; Parasuraman et al., 2005 ; Bauer et al., 2006 ; Collier et Bienstock, 2006) ou bien en termes de coûts (Ha et Stoel, 2009). Or, la livraison n'est pas qu'une question de temps ou d'argent (Collier et Bienstock, 2006). Le processus peut être organisé selon différentes options et devrait répondre aux questions « où », « quand » mais surtout « comment ». Cette réflexion est particulièrement importante dans le secteur du luxe où les éléments symboliques sont tout aussi importants que les éléments fonctionnels. Une exploration de la représentation de la livraison dans une approche logisticienne et de son évolution pourrait permettre d'approfondir cette question.

\section{D'une logistique fonctionnelle à une logistique expérientielle}

Pendant longtemps, la logistique a été appréhendée comme une recherche du zéro défaut, où aucun dysfonctionnement ne devait intervenir sur la chaîne, de la production à la livraison au client final. Les objectifs étaient d'obtenir un avantage concurrentiel durable grâce à une parfaite maîtrise des flux de produits, tout en optimisant les coûts, les délais et la réactivité (Paché, 2006). Une recherche de performance et d'excellence a ainsi longtemps été au cœur de la logistique (Mentzer et Bobbitt, 2004).

Mais depuis quelques années, nous assistons à une réelle évolution du métier et des fonctions qui lui sont associées, allant vers une logique « zéro ennui » (Badot et Paché, 2007). L'objectif est de ne plus considérer uniquement la vision rationnelle de la logistique mais d'en développer une approche davantage « expérientielle ». Certains parlent du besoin de ré-enchanter la logistique et des différents moyens pouvant être utilisés dans la distribution physique (Rouquet et Paché, 2017).

L'évolution de la fonction logistique a également conduit à la naissance de nouveaux acteurs (Baron, 2014). Les prestataires de services logistiques (PSL) se sont particulièrement développés afin de s'adapter aux nouveaux modes de consommation (Fulconis et al., 2011). L'avènement du e-commerce a notamment impacté l'ensemble de la chaîne logistique des marques (Kessous, 2001). La vente en ligne a entraîné un accroissement considérable des flux de colis en zone urbaine (Ducret, 2012). Elle implique de nouvelles contraintes et de nouveaux coûts pour les entreprises, particulièrement lors du « dernier kilomètre », regroupant les moyens utilisés pour acheminer les produits sur la dernière portion du parcours vers l'utilisateur final (Lacroux, 2018). Ce «dernier kilomètre » suppose aussi une hausse des échanges entre les clients et les marques (Kessous, 2001). Les prestataires de services logistiques et notamment les conducteurs-livreurs sont de plus en plus en interaction avec les consommateurs (Jacquemier-Paquin et al., 
2018). Toutefois, en confiant cette gestion du dernier kilomètre à des prestataires externes, un risque de dégradation du service rendu est à craindre (Lacroux, 2018). Cette dernière étape devrait alors être davantage contrôlée par les marques si elles ne veulent pas que cette nouvelle proximité nuise à leur image.

Le passage d'une logistique fonctionnelle à une logistique expérientielle constitue donc un véritable bouleversement pour les marques dans le sens où une gestion plus servicielle et personnalisée devrait être mise en œuvre, notamment lors de la livraison. La livraison implique de nouvelles difficultés mais peut, potentiellement, représenter un avantage concurrentiel saillant dans le cadre de la vente en ligne. Cela pourrait être le cas dans le luxe où les valeurs expérientielles sont propres au secteur. Toutefois, il est possible de s'interroger sur la diversité des attentes des consommateurs de luxe. Au regard de la théorie de la dualité du luxe, les motivations lors d'un achat sont multiples et les clients pourraient désirer des formes variées de livraison selon qu'ils aient une logique de consommation personnelle ou interpersonnelle. C'est pourquoi cette recherche met en œuvre une double méthodologie dont les objectifs sont : 1) d'étudier les attentes de consommateurs de luxe face à la livraison et 2) de les confronter aux pratiques des marques de luxe, afin d'en définir les contours et d'en déduire des contributions théoriques et managériales (voir l'encadré méthodologique). Les deux études menées analysent le discours des consommateurs et des marques en s'appuyant sur la sémiotique structurale. La sémiotique structurale est une théorie du sens et des procédures d'analyse permettant de décrire des systèmes de signification (Floch, 1990) et est particulièrement pertinente lorsqu'il s'agit d'étudier le sens profond des discours (Floch, 1990 ; Courtès, 1991).

\section{MÉTHODOLOGIES}

\section{1) Une analyse qualitative basée sur des entretiens de consommateurs}

L'étude s'appuie sur 18 entretiens ${ }^{3}$ individuels semi-directifs réalisés auprès d'hommes et de femmes, âgés de 25 à 71 ans, tous acheteurs et consommateurs de produits de marques de luxe (prêt-à-porter, maroquinerie, chaussures, joaillerie ou horlogerie), à des fréquences variées (acheteur occasionnel vs. régulier). Parmi eux, 7 ont déjà acheté en ligne des produits de marques de luxe (tableau 1). Un guide d'entretien semi-structuré est utilisé. Il se focalise sur : 1) la représentation du luxe (définition, fréquence et type d'achat), 2) le magasinage en boutique (expérience vécue, attentes en termes d'expérience) et 3) les perceptions face au e-commerce et à la livraison dans le luxe (expérience vécue ou souhaitée, façon dont la procédure d'achat est effectuée, dont le produit est livré). Les discours des consommateurs ont ensuite fait l'objet d'une analyse thématique catégorielle, utilisant une grille de lecture sémiotique basée sur l'opposition sémantique « luxe pour soi (personnel) - luxe pour les autres (interpersonnel) ». Un carré sémiotique est construit et permet d'affiner les analyses en

3. L'échantillon, composé de 18 consommateurs de luxe, répond au critère de saturation sémantique. 
faisant passer de 2 à 4 le nombre de profils de consommation du luxe, grâce à 3 types de relation (figure 1) : 1) la relation de contrariété ou d'opposition sémantique (lignes horizontales), 2) la relation de contradiction (lignes obliques) correspondant à une négation et 3) la relation de complémentarité (lignes verticales) correspondant à l'opération d'assertion (Floch, 1990). Les 4 profils sont : 1) le luxe pour soi valorisant le plaisir et l'émotion, 2) pour les autres valorisant la distinction sociale, 3) la négation du luxe pour les autres valorisant l'intimité, et 4) la négation du luxe pour soi valorisant le conformisme.

\section{2) Une analyse sémiotique visuelle structurale des marques de luxe}

Cette étude s'appuie sur une analyse sémiotique structurale visuelle de marques de luxe et plus spécifiquement de leurs pages de commandes de produits sur leurs sites Internet.

Dans un premier temps, l'analyse s'est portée sur la haute horlogerie. Le choix du secteur est lié aux réticences particulièrement importantes des marques horlogères à développer le ecommerce. Les sept marques partenaires de la Fondation de la haute horlogerie pratiquant de la vente en ligne (sur les 26 partenaires existants) ont été étudiées : Cartier, Hermès, Jaegger LeCoultre, Louis Vuitton, Montblanc, Piaget et Van Cleef \& Arpels (VCA). Des corpus de communication rassemblant des impressions d'écran des pages de commande et des procédures d'achat en ligne ont été créés. Des simulations d'achat ont été effectuées (prix des montres entre $3000 €$ et $15000 €$ ). Les discours ont été étudiés grâce à une grille d'analyse permettant d'identifier les signes récurrents des visuels (les signifiants) et leurs significations (les signifiés), prenant en compte les messages plastiques, figuratifs et linguistiques. Dans un second temps, des marques de prêt-à-porter de luxe ont complété les premières analyses. L'objectif est d'étudier si les discours de livraison mis en évidence dans le secteur de la haute horlogerie se retrouvent pour d'autres produits ayant un niveau de luxe différent ${ }^{4}$. Les trois marques qui sont les mieux valorisées dans le classement Interbrand 2018, dont le métier d'origine est le prêt-à-porter et qui pratiquent la vente en ligne pour les gammes de vêtements ont été prises en compte : Burberry, Dior et Gucci. La même procédure que pour les marques horlogères a été utilisée.

\section{III - ANALYSE DES ATTENTES DES CONSOMMATEURS DE LUXE VIS-À-VIS DE LA LIVRAISON}

Les discours des consommateurs de luxe interrogés sont analysés sur la base du carré sémiotique opposant « luxe pour soi (personnel) - luxe pour les autres (interpersonnel) ». Ils sont ensuite discutés selon les composantes fonctionnelles et expérientielles de la logistique.

\footnotetext{
4. Trois niveaux de luxe sont définis dans la littérature : le luxe accessible (faisant références aux catégories des parfums et des cosmétiques), le luxe intermédiaire (faisant référence aux accessoires et à la mode) et le luxe inaccessible (faisant référence à la joaillerie et à l'horlogerie) (De Barnier et al., 2012).
} 
Tableau 1 - Caractéristiques des individus interrogés

\begin{tabular}{|c|c|c|c|c|}
\hline Individu & Sexe & Âge & $\begin{array}{l}\text { Type de } \\
\text { consommateurs }\end{array}$ & $\begin{array}{l}\text { Achat en ligne } \\
\text { de marques de } \\
\text { luxe }\end{array}$ \\
\hline 1 & Homme & 35 & Régulier & Oui \\
\hline 2 & Homme & 38 & Régulier & Oui \\
\hline 3 & Homme & 29 & Régulier & Non \\
\hline 4 & Femme & 40 & Occasionnel & Non \\
\hline 5 & Homme & $\mathrm{NC}$ & Régulier & Oui \\
\hline 6 & Femme & 33 & Occasionnel & Oui \\
\hline 7 & Femme & 71 & Occasionnel & Non \\
\hline 8 & Homme & 40 & Occasionnel & Non \\
\hline 9 & Homme & 38 & Régulier & Non \\
\hline 10 & Femme & 55 & Occasionnel & Non \\
\hline 11 & Femme & 49 & Régulier & Non \\
\hline 12 & Homme & 35 & Régulier & Non \\
\hline 13 & Femme & 44 & Régulier & Non \\
\hline 14 & Femme & 27 & Régulier & Oui \\
\hline 15 & Femme & 33 & Régulier & Non \\
\hline 16 & Homme & 52 & Régulier & Oui \\
\hline 17 & Homme & 27 & Régulier & Oui \\
\hline 18 & Homme & 39 & Régulier & Non \\
\hline
\end{tabular}

\section{La livraison selon les quatre logiques de consommation du luxe}

\section{Luxe pour soi (personnel) : le plaisir et l'émotion}

Parmi les personnes interrogées, certains font référence au plaisir personnel de l'achat de produits de marques de luxe (« un certain plaisir de vivre » - indiv 10 ; «c'est épicurien... les grands plaisirs de la vie »
- indiv 16 ; « le plaisir » - indiv 8 et 9). Il s'agit d'hommes et de femmes, âgés de 38 à 55 ans. Certains d'entre eux sont des consommateurs réguliers du luxe et d'autres des consommateurs occasionnels. Ce groupe concerne principalement l'achat de produits de luxe intermédiaire à inaccessible.

Concernant le e-commerce, seule une personne de ce groupe a déjà procédé à l'achat en ligne de produits de marques de 
Figure 1 - Carré sémiotique des représentations de la livraison dans le luxe

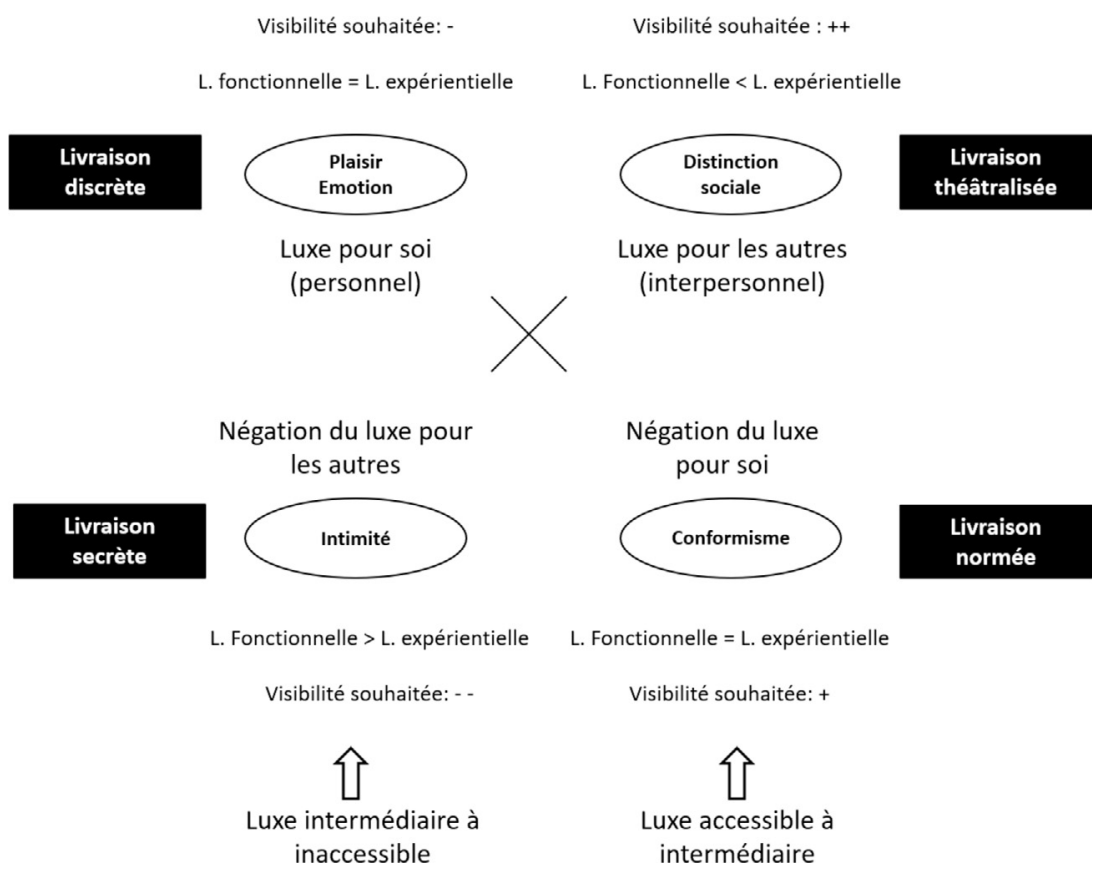

luxe. Les autres notent une incohérence entre les valeurs du luxe et Internet ( je trouve que c'est dénaturer le luxe »-indiv 10 ; « incompatibles car il n'y a dans l'ecommerce aucune expérience client qui est un des facteurs importants pour les produits de luxe » - indiv 9).

Plus spécifiquement en lien avec la livraison, dans le cas où ces individus se projetteraient sur un achat en ligne dans le luxe et, au-delà des attentes traditionnelles de tout achat en ligne (délais, sécurité) qu'ils jugent très importantes, ces consommateurs mettent en évidence le caractère hédonique que la livraison devrait avoir (« le plaisir d'ouvrir ses paquets » - indiv 10). Ils souhaiteraient que la livraison puisse instaurer une relation avec la marque («par quelqu'un de la marque, je n'ai pas envie que le facteur vienne me l'apporter » - indiv
8 ; « par un conseiller de la marque »indiv 9). En relation avec la composante hédonique désirée, ces consommateurs de luxe imaginent un emballage renforçant ce moment de découverte, insistant sur le caractère qualitatif des matériaux utilisés. Enfin, ils sont majoritairment contre les frais de livraison.

\section{Luxe pour les autres (interpersonnel) : la distinction sociale}

Ce groupe est représenté par des consommateurs réguliers du luxe, hommes et femmes, âgés de 29 à 44 ans. Pour ces personnes, le luxe est perçu comme un moyen de se distinguer des autres («ça évoque une réussite » - $\operatorname{indiv} 3$; «Un monde à part, accessible à une certaine catégorie de personnes »-indiv 13 ; «tout 
le monde ne peut pas les acheter $\gg-$ indiv 15). Ce groupe concerne principalement l'achat de produits de luxe accessible à intermédiaire.

Concernant l'e-commerce, la plupart des consommateurs de ce groupe note un risque de dilution des valeurs du luxe ( Je pense que le fait de se mettre sur Internet, c'est un manque de prestige »-indiv 3). Ils mettent également en avant une nette préférence pour l'achat traditionnel en boutique ( $\ll \mathrm{Je}$ préfère aller en magasin »-indiv 15).

Plus spécifiquement, lorsqu'ils évoquent le processus de livraison, ces consommateurs souhaiteraient que, au-delà des caractéristiques liées aux délais, à la rapidité et à la sécurité, considérées comme des éléments de base, ce moment sorte de l'ordinaire et représente au contraire l'extraordinaire qui, selon eux, définit ce qu'est le luxe. Ils souhaiteraient une livraison visible et démonstrative («qu'il sorte le tapis rouge » - indiv 2 ; « qu'une personne bien habillée vienne me livrer mon produit »- indiv 3 ; " pourquoi pas un charmant vendeur avec une coupe de champagne »- indiv 12). L'emballage devrait également refléter cette recherche d'exception et de mise en scène ostentatoire (« Je veux que mon produit soit livré dans un écrin »- indiv 2 ; «J'estime que quand j'achète un produit de luxe, je dois avoir un sac, un emballage avec le logo de la marque »- indiv 12).

\section{Négation du luxe pour les autres : l'intimité}

Ce groupe est représenté par des consommateurs qui mettent particulièrement en avant le fait qu'ils sont contre les démonstrations publiques du luxe ( Il ne faut pas qu'il y ait le logo dessus (le produit) »-indiv
7 ; « Tout ce que j'achète ce n'est vraiment pas pour montrer à mon voisin, mon amie »indiv 11). Ils évoquent la nécessité d'intimité dans les modes d'achat et de consommation du luxe («Souvent je cache que j'ai acheté quelque chose de luxueux (...) Oui, je n'ai pas envie que tout le monde me regarde »indiv 14). Il s'agit d'un homme et de trois femmes, âgés de 27 à 71 ans (avec une majorité de personnes de plus de 50 ans). Ce sont essentiellement des consommateurs réguliers du luxe et achetant des produits de luxe intermédiaire à inaccessible.

Ces consommateurs sont tous réticents à l'achat en ligne de produits de marques de luxe (« Non, c'est trop populaire internet (...) pour les produits de luxe pour moi c'est en dernier recours »- indiv 14).

Plus spécifiquement, lorsqu'ils évoquent la livraison, leurs attentes concernent principalement la protection des produits («Que l'article ait été bien préservé pendant son voyage »- indiv 7). Ces consommateurs spécifient clairement qu'ils sont contre toute démonstration de richesse lors de ce processus («Ah non pas de limousine. Moi j'aime bien être discrète (...) ; je n'ai pas besoin d'acheter un truc où est inscrit la marque en grand. Au contraire, plus c'est discret et plus je trouve ça chic »- indiv 14 ; « Je n'ai pas envie de cérémonial »indiv 7 ; « une livraison discrète, sécurisée, avec des sociétés spécialisées comme DHL, UPS ou autre »- indiv 5). L'emballage des produits lors de la livraison doit être conçu pour protéger avant tout les objets achetés ( «Il faut quand même un bon emballage, quelque chose de sécurisé » - indiv 7 ; « avec un petit livret de présentation de la marque et dans certains cas, un certificat d'identification »- indiv 14). Enfin, à la différence des autres groupes, ces personnes 
acceptent facilement l'idée de payer pour être livrées.

\section{Négation du luxe pour soi : le conformisme}

Pour ce groupe, le luxe est considéré comme un moyen de se conformer à un groupe ( « Pour moi le luxe c'est surtout un statut que tu as dans notre société, l'appartenance à un groupe et la manière dont que tu es perçu par les gens de ta profession »-indiv 1). Il est représenté par une consommation régulière et d'un niveau plutôt accessible.

La vente en ligne n'est pas perçue comme particulièrement cohérente ou incohérente dans le luxe (« Ça dépend de la catégorie du produit »- indiv 1).

Concernant les attentes face à la livraison dans le luxe, les standards de qualité (ponctualité, facilité) apparaissent comme des critères importants. En complément, la livraison doit être personnalisée et haut de gamme («J'aimerais être servi par coursier ; c'est-à-dire une personne qui viendrait délivrer mon produit et qui prendrait le temps de le faire »-indiv 1). Une attention particulière doit être donnée à l'emballage des produits, tant du point de vue de sa qualité que de son esthétique («Une bonne présentation du produit »-indiv 1). La livraison doit correspondre aux codes du luxe. Enfin, les frais de transport sont acceptés selon le prix de la commande.

\section{La livraison selon les dimensions fonctionnelle et expérientielle de la logistique}

L'analyse des entretiens menés a mis en évidence les attentes des individus selon quatre logiques de consommation. Celles-ci peuvent également être appréhendées en considérant les composantes fonctionnelles et expérientielles de la logistique (Badot et Paché, 2007 ; Rouquet et Paché, 2017). Un autre critère de différenciation émerge des analyses : il s'agit de la visibilité potentielle de la livraison. Les résultats sont synthétisés ci-après puis schématisés sur un carré sémiotique récapitulant les principales caractéristiques de chaque modèle de livraison (figure 1).

- Pour les consommateurs ayant une vision personnelle de la consommation du luxe (luxe pour soi), la livraison lors d'un achat en ligne devrait avoir tout autant une composante fonctionnelle (liée au respect des délais, à la praticité) qu'expérientielle (liée au plaisir et à l'émotion). Elle devrait être effectuée dans une sphère privée avec une faible visibilité potentielle. Elle est nommée « discrète ».

- Pour les consommateurs ayant une vision interpersonnelle, la composante expérientielle de la livraison (liée à une mise en scène de la domination sociale) est prédominante, bien que les standards classiques de qualité doivent être respectés. De plus, la livraison devrait être particulièrement visible aux yeux des autres. Elle est nommée «théâtralisée ».

- Pour les consommateurs étant dans une négation du luxe pour les autres, la composante fonctionnelle (liée essentiellement à la notion de protection et de sécurité) serait plus importante que la composante expérientielle (liée au plaisir). De plus, la livraison devrait être la plus discrète possible. Elle est nommée « secrète ».

- Enfin, pour les consommateurs étant dans une négation du luxe pour soi, tout comme 
les consommateurs ayant une vision personnelle, la composante fonctionnelle (liée au respect des délais, à la praticité) et la composante expérientielle de la livraison (liée au besoin de conformité et d'appartenance à un groupe ainsi qu'au besoin de respecter les conventions) sont toutes aussi importantes. Par contre, à la différence du premier groupe, ces consommateurs souhaiteraient que la livraison soit visible. Elle est nommée « normée ».

\section{IV - ANALYSE DES DISCOURS DES MARQUES DE LUXE SUR LA LIVRAISON}

Le discours linguistique et visuel des marques de haute horlogerie et de prêt-àporter de luxe peut être étudié selon les deux composantes de la livraison : fonctionnelle (praticité, sécurité, frais de livraison, délais et ponctualité) et expérientielle (esthétique, hédonisme) (Badot et Paché, 2007 ; Rouquet et Paché, 2017).

\section{Le discours sur la livraison fonctionnelle : sécurité et ponctualité}

Les marques étudiées insistent sur les récits de la sécurité et de la ponctualité.

La sécurité est liée à la protection des objets précieux achetés en ligne, de leur transport jusqu'au transfert de propriété («Cartier assure les achats pendant la durée de leur transit jusqu'à ce qu'ils vous soient livrés. Nous exigeons la signature d'un adulte confirmant la réception de chaque produit Cartier livré, moment auquel la responsabilité des marchandises achetées vous revient » Cartier ; «La Maison prête une attention particulière aux commandes passées sur la boutique en ligne Van Cleef \& Arpels » VCA). L'expression «Livraison sécurisée » est particulièrement répétée sur l'ensemble des sites Internet. Ce discours passe également par la spécification de l'utilisation d'un partenaire spécialisé en transport, comme UPS Express Saver, FedEx ou Chronopost.

La ponctualité est liée au respect du délai prévu (« Nous ferons tout notre possible pour respecter les délais de livraison estimés à compter de la date de confirmation de votre commande » Cartier ; « Nous ferons tout ce qui est raisonnablement possible pour assurer une livraison par un transporteur dans les délais de livraison estimés » VCA ; « 2 jours ouvrés environ/offerts » Louis Vuitton). Elle peut également passer par la précision des dates («Jour estimé de livraison : le lundi X juin 20XX» Piaget ; « La date estimée de livraison est : le lundi $\mathrm{X}$ juin 20XX » VCA) ou celui de la rapidité (« livré à domicile entre18 et $20 \mathrm{~h}$, pour toute commande passée avant $13 \mathrm{~h}$ le jourmême » Louis Vuitton).

Au-delà de ces deux éléments, le récit autour de la composante fonctionnelle de la livraison fait référence aux frais que celle-ci peut engendrer. Certaines marques prônent la gratuité (Burberry, Jaegger LeCoultre, Piaget) et ce quelle que soit la rapidité de la livraison. D'autres, comme Dior, Gucci, Van Cleef \& Arpels, Montblanc et Cartier, proposent la gratuité pour une livraison standard mais imposent des frais croissants en fonction de la rapidité de la livraison. Enfin, Hermès et Louis Vuitton mettent en avant la gratuité de la livraison mais proposent un service davantage personnalisé payant (l'utilisation d'un coursier pour Hermès ou une livraison dite « Premium » 
pour Louis Vuitton, sans toutefois donner plus de précisions).

\section{Le discours sur la livraison expérientielle : la mise en beauté des produits}

À la différence du discours sur la composante fonctionnelle de la livraison, celui sur la composante expérientielle est beaucoup plus limité et n'est pas présent pour toutes les marques étudiées. Lorsqu'il est existant, il met en avant la présentation des produits des marques de luxe achetés en ligne et passe essentiellement par l'emballage.

Certaines marques utilisent un discours linguistique en décrivant le contenu du colis (« Dans chaque emballage, vous trouverez, outre votre produit Cartier acheté : 1) tous les accessoires annexes, le cas échéant, 2) une carte de remerciement ;
3) le dossier de documentation comprenant le manuel du produit et le certificat d'authenticité ainsi que la garantie internationale (...) » Cartier et Piaget). D'autres insistent sur la composante sensorielle de l'emballage («Tous nos paquets sont magnifiquement emballés dans leur coffret Van Cleef \& Arpels exclusif. Le coffret Van Cleef \& Arpels et les emballages spéciaux peuvent être précieux (...). Toutes nos créations sont livrées délicatement emballées dans un écrin cadeau exclusif à notre boutique en ligne » VCA), et font référence à leur identité visuelle (« Tous les objets commandés sont livrés dans une boîte orange nouée de bolduc » Hermès).

Le récit de mise en beauté passe également par le discours visuel. La présentation des boîtes aux couleurs mythiques de chaque marque permet de mettre en valeur les dimensions sensorielles (figure 2). Enfin,

Figure 2 - Discours visuel des marques sur l'emballage (récit de l'esthétique)

Van Cleef \& Arpels

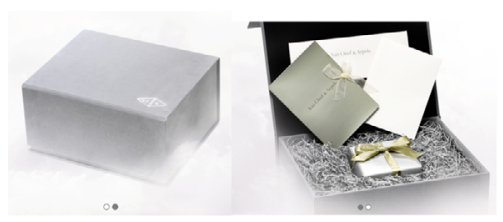

Hermès

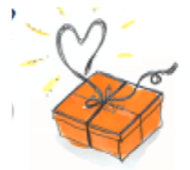

Burberry

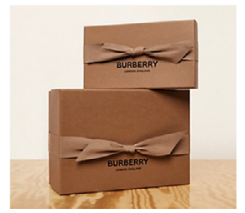

Cartier

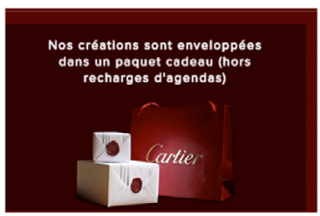

Piaget

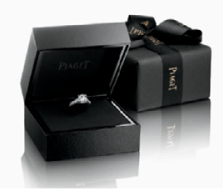

Dior

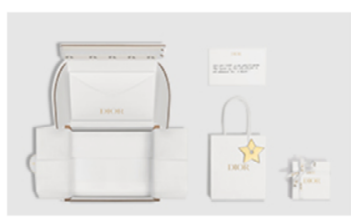


l'imaginaire des consommateurs à propos d'une livraison esthétisée peut être suscité. C'est le cas de Louis Vuitton qui, en proposant une livraison « Premium », promet une "une prestation d'exception », sans pour autant détailler son contenu.

L'analyse des discours des marques de luxe étudiées montre une prédominance de la composante fonctionnelle de la livraison. Les récits sur la ponctualité, les délais, la sécurité et la gratuité sont présents pour les sept marques d'horlogerie de luxe et pour les trois marques de prêt-à-porter de luxe. Par contre, le discours sur la composante expérientielle est beaucoup plus rare. Toutes les marques ne le développent pas sur leur site (comme par exemple Jeagger LeCoultre ou Montblanc) et lorsqu'il est présent, il n'est parfois évoqué que sous certaines conditions (par exemple en cliquant sur l'option « cadeau »).

\section{V - LE DÉCALAGE ENTRE LES ATTENTES DES CONSOMMATEURS ET LE DISCOURS DES MARQUES}

L'analyse des discours linguistiques et visuels utilisés pour exprimer la livraison sur les sites Internet des marques d'horlogerie et de prêt-à-porter de luxe étudiées, mise en rapport avec les résultats de l'étude sur les attentes des consommateurs de luxe, montre un décalage important et une non prise en compte de l'ensemble des attentes des profils de consommation (figure 3 ).

- Sept marques (Burberry, Cartier, Dior, Gucci, Hermès, Piaget et Van Cleef \& Arpels) font référence à la composante

Figure 3 - Confrontation du discours des consommateurs de luxe et des marques de luxe face à la livraison

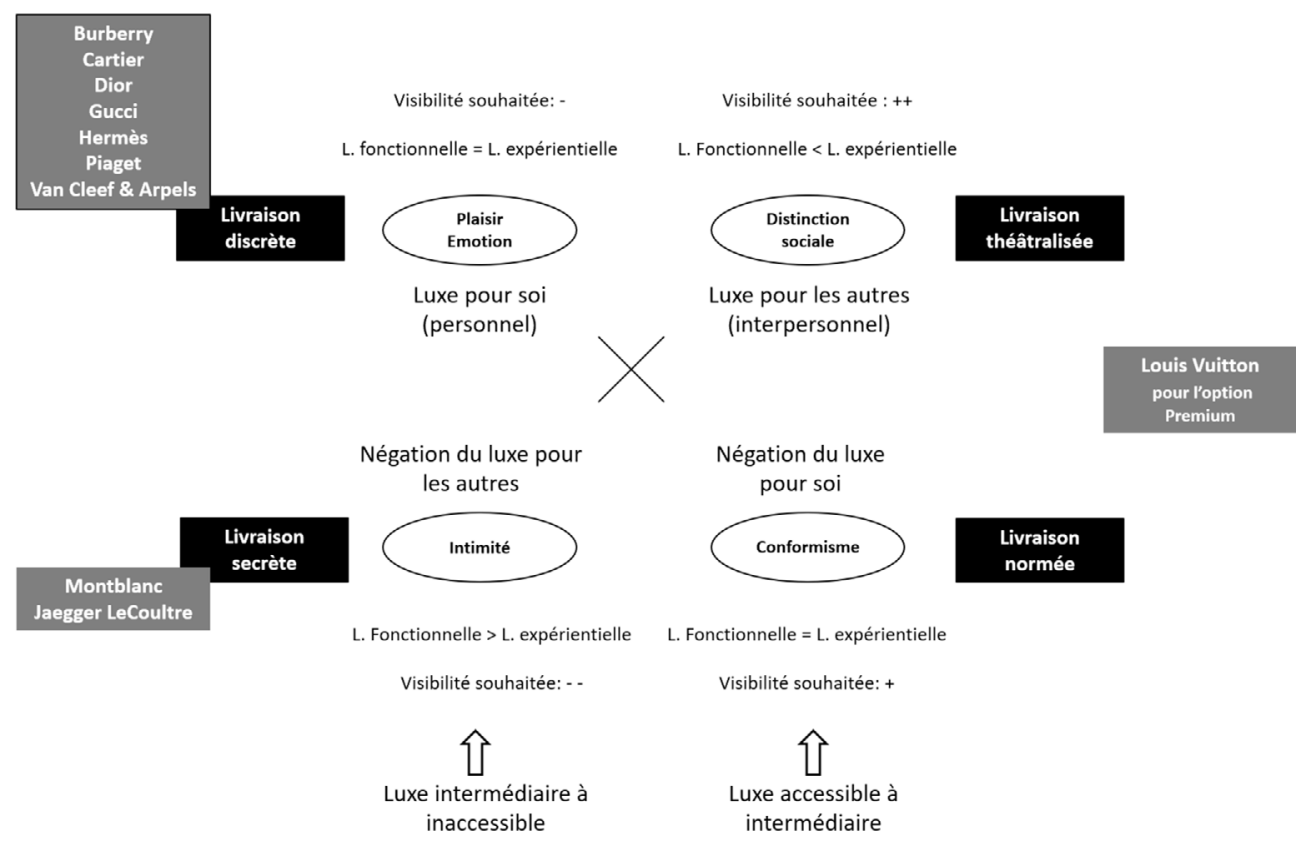


fonctionnelle de la livraison et évoquent de façon limitée l'esthétique de la livraison. Cette proposition de livraison pourrait correspondre aux clients ayant une logique personnelle du luxe (pour soi) si le discours sur la composante expérientielle était davantage développé.

- Deux marques (Montblanc et Jaegger LeCoultre), en se focalisant sur les aspects fonctionnels de la livraison, se rapprochent de la vision des consommateurs qui considèrent la négation du luxe pour les autres et valorisent les aspects sécuritaires du processus.

- Seule une marque (Louis Vuitton) évoque une livraison d'exception (livraison premium) qui pourrait potentiellement valoriser la dimension expérientielle liée à l'extraordinaire. Toutefois, aucun détail n'est donné sur la façon dont la livraison est effectuée. Si l'on considère cette option, la livraison proposée par Louis Vuitton pourrait correspondre aux attentes des consommateurs ayant une vision interpersonnelle du luxe (pour les autres) ou une négation du luxe pour soi.

\section{VI - DISCUSSION DES RÉSULTATS}

La double méthodologie de la recherche a permis d'identifier quatre modèles de livraison souhaités par les consommateurs de produits de luxe. Ceux-ci sont liés aux logiques de consommation du luxe (Vigneron et Johnson, 2004) et reflètent une importance différente des composantes fonctionnelles et expérientielles de la logistique (Badot et Paché, 2007 ; Rouquet et Paché, 2017). Ils sont également fonction d'une caractéristique complémentaire ressortie lors des analyses: la visibilité souhaitée de la livraison. Ces modèles sont :
- La livraison discrète (luxe pour soi) : importance identique des composantes fonctionnelles et expérientielles, avec une faible visibilité ;

- La livraison théâtralisée (luxe pour les autres) : prédominance de la composante expérientielle, avec une très forte visibilité ; - La livraison secrète (négation du luxe pour les autres): prédominance de la composante fonctionnelle, avec une très faible visibilité ;

- Et la livraison normée (négation du luxe pour soi) : importance identique des composantes fonctionnelles et expérientielles, avec une forte visibilité.

\section{Contributions théoriques de la recherche}

Plusieurs contributions théoriques découlent des résultats de cette recherche.

Tout d'abord, cet article apporte une pierre supplémentaire aux travaux qui soulignent que la logistique ne doit pas être vue uniquement sous un angle fonctionnel mais aussi en intégrant une composante hédonique et expérientielle (Badot et Paché, 2007 ; Rouquet et Paché, 2017). Grâce à l'analyse des perceptions des consommateurs, il a été montré que les aspects pratiques (délais, ponctualité, frais de livraison) ne suffisent plus. Les attentes se tournent également vers le plaisir du moment vécu lorsque les individus reçoivent leurs produits. Parmi les quatre modèles de livraison identifiés dans cet article, trois valorisent la composante expérientielle. Cela est particulièrement le cas de la livraison théâtralisée dont la mise en scène est perçue comme plus importante que les questions relatives à la fonctionnalité de celle-ci. Ce point peut être mis en 
relation avec l'évolution de la distribution de manière plus globale. Depuis plus d'une vingtaine d'année, les boutiques ne se pensent plus uniquement comme des lieux transactionnels. Elles y associent une mise en scène de l'assortiment et la construction d'une atmosphère élaborée, permettant la théâtralisation de l'offre et le développement d'un marketing relationnel (Filser, 2001). La théâtralisation de la livraison constitue donc une suite logique à l'évolution qu'a connu, il y a quelques temps, la distribution physique avec les points de vente.

De plus, cet article est le premier à s'intéresser à la question de la livraison dans le luxe. Il répond aux précédentes recherches indiquant que des travaux spécifiques doivent être dédiés à ce secteur (Bastien et Kapferer, 2008). L'utilisation de la théorie de la dualité du luxe a permis de mieux appréhender les attentes des consommateurs de luxe et de proposer quatre modèles différents de livraison souhaitables pour les consommateurs. Ces résultats rejoignent les travaux antérieurs mettant en évidence la nécessité de développer des stratégies différentes selon les profils de consommateurs de luxe (Bastien et Kapferer, 2008 ; Veg-Sala et Geerts, $2015 b$ ) et selon les niveaux de luxe (De Barnier et al., 2012), également présents dans cette recherche.

Par ailleurs, la double approche fonctionnelle et expérientielle de la livraison dans le luxe a pour conséquence une impossibilité d'utiliser en l'état les échelles de mesure existantes sur la qualité de service en ligne. En effet, les principaux outils de mesures de la qualité de service en ligne ne donnent que très peu de place à la livraison et ne l'envisagent qu'en termes de ponctualité ou de coûts (Wolfinbarger et Gilly, 2003 ; Parasuraman et al., 2005 ; Bauer et al., 2006 ; Collier et Bienstock, 2006 ; Ha et Stoel, 2009). De plus, les caractéristiques fonctionnelles de la livraison de ces échelles, liées à la rapidité, au délai et à la ponctualité de la livraison, ne correspondent pas à l'éthique du luxe, telle que définie par Lipovetsky et Roux (2003). L'éthique correspond au refus du tout-économique, impliquant non seulement l'absence de nécessité dans le luxe mais aussi l'absence d'une entière maîtrise sur le monde et donc sur le temps.

Enfin, d'un point de vue méthodologique, la création du carré sémiotique basé sur l'opposition sémantique «luxe pour soi (personnel) - luxe pour les autres (interpersonnel) » permet d'approfondir les logiques de consommation du luxe et de prendre en compte de façon plus précise les spécificités du luxe (Lipovetsky et Roux, 2003). Cet outil vient donc compléter la littérature sur le luxe et pourrait être utilisé dans d'autres recherches sur cette thématique.

\section{Contributions managériales de la recherche}

Au-delà des apports théoriques, les résultats de l'étude sur les attentes des consommateurs de luxe confrontés au discours des marques (de luxe) conduisent à avancer plusieurs recommandations pour les entreprises.

Premièrement, à travers la détermination des quatre modèles de livraison dans le luxe, il a été mis en évidence que la qualité perçue de cette étape lors d'un achat en ligne est primordiale et pourrait permettre d'atténuer certains freins face au e-commerce dans ce secteur en particulier. La composante 
fonctionnelle étant présente pour l'ensemble de ces modèles, la conceptualisation de la livraison devrait au minimum respecter les standards de qualité du luxe. Mais, à la différence de ce qu'il se pratique de nos jours, les marques doivent tenir compte du fait que les éléments fonctionnels ne sont plus suffisants pour plusieurs profils de consommateurs. La livraison théâtralisée et la livraison normée, actuellement absentes des processus de vente en ligne des marques de luxe, devraient être développées afin de répondre à l'ensemble des attentes des clients. Deuxièmement, concernant la mise en œuvre de la livraison, les managers doivent prendre en compte que les caractéristiques individuelles mises en évidence afin de distinguer les attentes des clients (logiques de consommation, visibilité souhaitée) sont difficilement appréhendables par les marques. Il est difficile de connaître leurs motivations vis-à-vis d'un achat dans ce secteur lorsque ces personnes, de chez elles, de leur travail ou de leur lieu de vacances, achètent pour la première fois. De ce fait, les maisons de luxe devraient donner la possibilité aux consommateurs de choisir entre plusieurs types de livraison et pas uniquement une livraison discrète ou secrète qui s'appuie sur des logiques de consommation tournées vers soi ou en opposition aux autres. Les deux autres modes de livraison (théâtralisée et normée) concer-nent des personnes qui ont d'autres optiques de consommation et qu'il ne faut pas négliger. Si les marques de luxe ne répondent pas à leurs attentes sur ce point, alors qu'il s'agit d'un des freins les plus importants identifiés pour l'achat en ligne dans le secteur du luxe, les marques risquent de perdre un grand nombre de ventes potentielles ou bien de ne pas satisfaire ses clients et in fine de diluer leur image de marque. Plus particulièrement, grâce à la prise en compte des niveaux de luxe des produits étudiés, il est recommandé de proposer une livraison théâtralisée ou normée pour les marques dites de luxe accessible ou intermédiaire.

Troisièmement, les marques de luxe devraient réfléchir à la façon d'opérationnaliser les livraisons théâtralisées ou normées. La création de scénarios devrait être incluse dans les stratégies de vente en ligne. Plusieurs pistes peuvent être évoquées ici : utilisation d'une voiture de luxe, utilisation de gants blancs, coupe de champagne offerte... (les coûts pouvant varier et s'adapter à chaque marque et à chaque vente). Les marques pourraient gérer ce type de livraison en interne (en utilisant par exemple les conseillers de vente et en les rendant davantage mobiles) ou bien faire appel à des prestataires de services logistiques spécialisés dans le secteur du haut de gamme comme Kronos ou Excelcourses. Ces PSL, ou les PSL plus généralistes, pourraient par ailleurs utiliser les résultats de cette recherche afin de préciser leurs offres, ou d'en développer de nouvelles pour gagner de nouvelles parts de marché dans un secteur où la croissance en ligne est estimée à $22 \%$ par an ${ }^{5}$.

Quatrièmement, au-delà de la conception des différents modes de livraison possibles,

5. Bain \& Company (2018). «Luxury Goods Worldwide Market Study », Fall-Winter https://www.bain.com/ contentassets/8df501b9f8d6442eba00040246c6b4f9/bain_digest_luxury_goods_worldwide_market_study_fall_winter_2018.pdf 
les marques de luxe devraient particulièrement soigner leur présentation en ligne. Il ne s'agit pas uniquement de la mise en œuvre mais aussi du discours linguistique et visuel qu'elles doivent développer sur les pages de commande. Elles devraient maintenir leurs récits sur les éléments fonctionnels de la livraison mais en les rendant moins visibles et dominants. Les consommateurs de luxe ont toujours besoin d'être rassurés sur la façon dont les produits vont leur être délivrés. Mais pour maintenir les valeurs du luxe et leur désirabilité, les marques pourraient rendre davantage visible le discours sur la composante expérientielle. Pour les livraisons discrètes, les marques pourraient mettre davantage en avant l'esthétique de la livraison avec notamment la mise en beauté des produits et leur emballage. Pour les livraisons théâtralisées ou normées, un descriptif de la procédure avec un texte et quelques photos pour susciter l'envie pourraient être insérés. Bien évidemment, comme ces livraisons devraient être adaptées à chaque commande, les marques ne devraient pas être trop précises sur leur contenu. Il faudra garder une part de mystère.

Cinquièmement, concernant les frais de livraison, et à la différence de ce que pratique la majorité des marques de luxe aujourd'hui, ceux-ci devraient complètement être absorbés par les maisons de luxe quand il s'agit de produits de luxe intermédiaire à inaccessible, que cela soit pour une livraison discrète, secrète, théâtralisée ou normée. En effet, à partir du moment où certains consommateurs sont formellement contre, il est important que ces frais ne deviennent pas un frein à l'achat en ligne. Cette décision de ne pas faire payer les livraisons doit être prise en compte dans le business model global de l'entreprise et pourra donc avoir des répercussions sur les prix des produits (tout comme cela est déjà le cas avec l'intégration des coûts liés à la gestion des boutiques). Concernant le luxe accessible, des frais pourraient être facturés $\mathrm{du}$ fait de l'impression d'un achat plus classique en ligne. Toutefois, ils devront être limités et justifiés par la qualité des prestations délivrées.

Enfin, un effort tout particulier, comme en boutique, doit être porté sur l'emballage des produits afin qu'il reflète la qualité et les valeurs du luxe et ce pour n'importe quel niveau d'achat de luxe (de l'accessible à l'inaccessible). Les marques de luxe devraient créer un packaging spécifique pour le transport et la livraison. Le choix des boîtes sera particulièrement important tout comme l'ensemble des matériaux utilisés pour protéger et sublimer les produits. Le moment où les clients ouvrent et découvrent leurs produits doit être multisensoriel. Il ne faudra donc pas négliger les sensations au toucher ou encore les odeurs et parfums.

\section{VII - POUR ALLER PLUS LOIN...}

Cet article n'est pas exempt de limites qui conduisent à de nouvelles pistes de recherche. L'analyse qualitative menée sur les consommateurs de luxe a eu pour objet de mieux comprendre leurs attentes face à la livraison pour l'achat de produits de marques de luxe. Cette étude s'est basée sur un échantillon hétérogène mais s'est focalisée sur trois pays francophones. Les visions du luxe variant d'une région du monde à l'autre, nous pensons que les résultats pourraient être différents aux États-Unis, au MoyenOrient ou encore en Asie. Les analyses menées ici pourraient être effectuées dans 
d'autres zones géographiques seraient donc nécessaires.

Concernant le discours des marques de luxe, la recherche s'est appuyée sur l'horlogerie et le prêt-à-porter pour mieux appréhender les pratiques mises en œuvre à ce jour. L'étude de ces deux secteurs a permis de vérifier que les résultats ne dépendaient pas des produits analysés. Il serait toutefois intéressant de poursuivre la démarche en intégrant de nouvelles marques mais surtout en ayant un suivi longitudinal pour identifier les évolutions. Une réflexion serait égale-ment à mener sur les secteurs pouvant être tout autant ou partiellement sensibles à la composante expérientielle de la livraison. La livraison étant le moment où une interaction physique est possible entre la marque et le client, une piste de recherche pourrait être l'optimisation de cette rencon-tre. Il serait alors possible d'utiliser la littérature sur la co-création ou sur la place du client dans l'organisation.

Enfin, la réflexion autour de la livraison dans le luxe peut amener de nouvelles recherches à se questionner sur le développement durable (Kessous et al., 2015), sur le tri des déchets et des emballages des colis (Monnot et al., 2014). La logistique est devenue un pilier dans l'établissement de procédures respectueuses de l'environnement et pourrait être un véritable atout pour les marques de luxe. Celles-ci sont considérées comme ayant les actifs intangibles, symboliques et immatériels les plus importants (en comparaison avec les autres marques) et le fait d'y ajouter une dimension sociale ou environnementale pourrait améliorer et renforcer l'imaginaire que les consommateurs leur associent. De futures recherches pourraient se consacrer à cette thématique, en incluant le transport et les emballages.

\section{BIBLIOGRAPHIE}

Badot O. et Paché G. (2007). « Une logistique expérientielle pour la firme de distribution : du « zéro défaut » au « zéro ennui » ", Management \& Avenir, vol. 1, n 11, p. 11-28.

Baron M.-L. (2014). «Les mutations de la logistique de distribution », La logistique : ses métiers, ses enjeux, son avenir, Brun D. et Guérin F., Éditions EMS, coll. « Management \& Société ».

Bastien V. et Kapferer J.-N. (2008). Luxe oblige, Eyrolles Éditions d'organisation, Paris.

Bauer H.H., Falk T. et Hammerschmidt M. (2006). "ETransQual: A transaction process-based approach for capturing service quality in online shopping", Journal of Business Research, vol. 59 , p. 866-875.

Bourdieu P. (1979). La distinction : critique sociale du jugement, Éditions de Minuit, Paris.

Catry B. (2007). « Le luxe peut être cher, mais est-il toujours rare ?», Revue française de gestion, vol. $33, \mathrm{n}^{\circ} 171$ p. $49-63$.

Collier J.E. et Bienstock C. (2006). "Measuring service quality in e-retailing", Journal of Service Research, vol. 8, n 3, p. 260-275

Courtès J. (1991). Analyse sémiotique du discours : de l'énoncé à l'énonciation, Éditions Hachette, Paris. 
Cristobal E., Flavian C. et Guinalıu M. (2007). "Perceived e-service quality (PeSQ): Measurement validation and effects on consumer satisfaction and web site loyalty", Managing Service Quality, vol. 17, no 3, p. 317-340

Dall'Olmo Riley F. et Lacroix C. (2003). "Luxury branding on the Internet: Lost opportunity or impossibility?”, Marketing Intelligence \& Planning, vol. 21, n 2, p. 96-104.

De Barnier V., Falcy S. et Valette-Florence P. (2012). "Do consumers perceive three levels of luxury? A comparison of accessible, intermediate and inacessible luxury brands", Journal of Brand Management, vol. 19, p. 623-636

Ducret R. (2012). «Livraison de colis et logistique urbaine : quelles recompositions de la messagerie en milieu urbain ?», Revue française de gestion industrielle, Association française de gestion industrielle, vol. 31, $\mathrm{n}^{\mathrm{o}} 3$, p. 29-48.

Filser M. (2001). «Le magasin amiral : de l'atmosphère du point de vente à la stratégie relationnelle de l'enseigne », Décisions Marketing, $\mathrm{n}^{\mathrm{o}}$ 24, p. 7-16.

Floch J.-M. (1990). Sémiotique, marketing et communication : sous les signes, les stratégies, Presse Universitaire de France (PUF).

Fulconis F., Paché G. et Roveillo G. (2011). La prestation logistique : origines, enjeux et perspectives, Editions EMS, coll. « Management \& Société ».

Granzin K.L. et Bahn K.D. (1989). “Consumer logistics: Conceptualization, pertinent issues and a proposed program for research", Journal of the Academy of Marketing Science, vol. 17, $\mathrm{n}^{\mathrm{o}} 1$, p. 91-101.

Ha S. et Stoel L. (2009). "Consumer e-shopping acceptance: Antecedents in a technology acceptance model”, Journal of Business Research, vol. 62, p. 565-571.

Jacquemier-Paquin L., Claye-Puaux S. et Jeanpart S. (2018). « La dimension "relation client" du métier de conducteur-livreur, un facteur d'engagement envers le métier », Management \& Avenir, vol. 2, $\mathrm{n}^{\mathrm{o}} 100$, p. 47-71.

Kastanakis M.N. et Balabanis G. (2012). "Between the mass and the class: Antecedents of the 'brandwagon' luxury consumption behaviour", Journal of Business Research, vol. 65, $\mathrm{n}^{\circ} 10$, p. 1399-1407.

Kessous A., Boncori A.-L. et Paché G. (2015). « Le transport durable dans la grande distribution - Quelle perspectives des consommateurs ? , Revue française de gestion, vol. 41, no 250 , p. 13-30.

Kessous E. (2001). « Le commerce électronique et la continuité de la chaîne logistique : De l'approvisionnement des sites à la livraison aux consommateurs », Réseaux, no 106/2, p. 103-133.

Lacroux A. (2018). « Le salarié, grand oublié de la 'logistique du dernier kilomètre' ? », Revue française de gestion, vol. 44, $\mathrm{n}^{\mathrm{o}} 277$, p. 155-158.

Lipovetsky G. et Roux E. (2003). Le luxe éternel : de l'âge du sacré au temps des marques, Gallimard, Paris. 
Liu X., Burns A.C. et Hou Y. (2013). "Comparing online and in-store shopping behavior towards luxury goods", International Journal of Retail \& Distribution Management, vol. 41, $\mathrm{n}^{\mathrm{o}} 11 / 12$, p. 885-900.

Mentzer S.M. et Bobbitt L. (2004). "Toward a unified theory of logistics", International Journal of Physical Distribution \& Logistics Management, vol. 34, n ${ }^{\circ}$ 8, p. 606-627.

Merk M. et Michel G. (2019). "The dark side of salesperson brand identification in the luxury sector: When brand orientation generates management issues and negative customer perception", Journal of Business Research, vol. 102, p. 339-352.

Okonkwo U. (2009). "Sustaining luxury brands on the Internet", Journal of Brand Management, vol. 16, $\mathrm{n}^{\mathrm{o}}$ 5-6, p. 302-310.

Paché G. (2006). « Concilier production au plus juste et agilité dans les chaînes logistiques étendues : un double éclairage opérationnel et stratégique », Gestion 2000, vol. 23, n ${ }^{\circ} 2$, p. 23-40.

Parasuraman A., Zeithaml V. et Malhotra A. (2005). "E-S-QUAL: A multiple-item scale for assessing electronic service quality”, Journal of Service Research, vol. 7, p. 1-21.

Rouquet A. et Paché G. (2017). "Re-enchanting logistics: The cases of pick-your-own farm and large retail stores", Supply Chain Forum: An International Journal, vol. 18, nº 1, p. 21-29.

Roux E. et Floch J.-M. (1996). « Gérer l'ingérable : la contradiction interne de toute maison de luxe », Décisions Marketing, no 9, p. 15-22.

Roux E., Tafani E. et Vigneron F. (2017). "Values associated with luxury brand consumption and the role of gender", Journal of Business Research, vol. 71, p. 102-113.

Shukla P. et Purani K. (2012). "Comparing the importance of luxury value perceptions in cross-national contexts", Journal of Business Research, vol. 65, n 10, p. 1417-1424.

Trocchia P. et Janda S. (2003). "How do consumers evaluate internet retail service quality?", Journal of Services Marketing, vol. 17, $\mathrm{n}^{\mathrm{o}}$ 3, p. 243-253

Veg-Sala N. et Geerts A. (2015a). «L'expérience de magasinage en boutique et en ligne pour les marques de luxe : Etude exploratoire sur les dimensions du concept », $31^{e}$ Congrès de l'Association française du marketing, Marrakech, vol. 20, 21-22 mai.

Veg-Sala N. et Geerts A. (2015b). « Le paradoxe de la distribution des marques de luxe face à l'e-commerce : proposition d'une typologie de consommateurs et de son évolution dans le temps », Décisions Marketing, n ${ }^{\circ}$ 80, p. 69-85.

Vigneron F. et Johnson L.W. (1999). "A review and a conceptual framework of prestigeseeking consumer behaviour", Academy of Marketing Science Review, www.amsreview.org/ articles/vigneron 01-1999.pdf.

Vigneron F. et Johnson T.W. (2004). "Measuring perception of brand luxury", Brand Management, vol. 11, $\mathrm{n}^{\mathrm{o}}$ 6, p. 484-506.

Wiedmann K.-P., Hennigs N. et Siebels A. (2007). "Measuring consumers' luxury value perception: A cross-cultural framework", Academy of Marketing Science, vol. 7, p. 1-21. 
Wolfinbarger M. et Gilly M. (2003). "eTailQ: Dimensionalizing, measuring and predicting etail quality", Journal of Retailing, vol. 79, p. 183-198

Wong N. et Ahuvia A.C. (1998). "Personal taste and family face: Luxury consumption in Confucian and Western societies”, Psychology and Marketing, vol. 15, n ${ }^{\circ}$ 5, p. 423-441.

Zeithaml V. (2002). "Service excellence in electronic channel”, Managing Service Quality: An International Journal, vol. 12, n ${ }^{\circ}$ 3, p. 135-139. 